\title{
COVID-19 Disease and Treatment of COVID-19
}

\author{
Youngah Choi* \\ Department of Internal medicine, Seoul Metropolitan Seobuk Hospital, Seoul 03433, Korea
}

*Corresponding author: Youngah Choi, Department of Internal medicine, Seoul Metropolitan Seobuk Hospital, Seoul 03433, Korea

\begin{tabular}{|c|c|}
\hline ARTICLE INFO & ABSTRACT \\
\hline Received: 慧 September 23, 2021 & $\begin{array}{l}\text { Citation: Youngah Choi. COVID-19 Disease and Treatment of COVID-19. Biomed J Sci \& } \\
\text { Tech Res 39(2)-2021. BISTR. MS.ID.006270. }\end{array}$ \\
\hline
\end{tabular}

\section{Introduction}

\section{Severe Acute Respiratory Syndrome Coronavirus 2(SARS-CoV-2)}

i. SARS-CoV-2 is a positive-stranded RNA virus with envelope, a kind of a 'Beta Corona Virus'.

ii. It is speculated that its RNA sequence and that of the bat coronavirus are more than $80 \%$ similar. There is a strong theory that the pangolin acted as an intermediate between bats and humans.

\section{Infection Route of COVID-19}

i. Although it is estimated that human-to-human transmission through droplets is the primary route of transmission, a number of research results suggesting the possibility of airborne transmission have recently been published.

ii. Although there are reports that SARS-CoV-2 was cultured in secretions such as feces or urine, it is not clear whether transmission through feces or urine can actually occur.

\section{Infectivity of COVID-19}

i. Summarizing the results of the studies which have so far been conducted, it seems that COVID-19 patients become contagious 2-3 days before the onset of symptoms. Infectivity usually lasts 5-7days after which it decreases.

Clinical Symptoms and Complications of COVID-19 Disease

i. Asymptomatic (50\%), mild-to-moderate $(40 \%)$, severe $(10 \%)$ including respiratory failure.

ii. Cough $(50 \%)$, high fever $\left(40 \%: 38^{\circ} \mathrm{C}\right.$ or higher), headache (35\%), dyspnea (30\%), sore throat (20\%), diarrhea (20\%), nausea and vomiting (15\%).

iii. Other symptoms include loss of smell, loss of taste, fatigue, decreased appetite, hemoptysis, dizziness, runny nose, nasal congestion, chest pain, conjunctivitis, skin changes, etc.

iv. Respiratory Complications: Lung fibrosis due to ARDS and pneumonia

v. Cardiovascular Complications: Elevated blood pressure, acute myocardial injury, arrhythmia, myocarditis, heart failure, acute coronary syndrome, atherosclerotic event and venous thromboembolism

vi. Acute Kidney Injury: Acute kidney injury due to high fever and dehydration and poor oral intake

vii. Gastrointestinal Complications: poor oral intake, indigestion, diarrhea, nausea and vomiting, elevated hepatobiliary function parameters and pancreatic enzyme levels. 
viii. Cytokine Release Syndrome: ARDS, sepsis, DIC, multiple organ failure pattern

ix. Nervous system-related symptoms and complications: Ischemic stroke, encephalitis, meningitis, impaired consciousness, Ataxia, convulsions, neuralgia.

\section{COVID -19 Disease Patterns and Severity Risk Factors in Patients with Pre-Existing Diseases}

i. Elderly patients with many underlying diseases are considered to have a high mortality rate because they cannot endure the symptoms and clinical manifestations itself of COVID-19 (high fever, high blood pressure, dehydration, breathing difficulty, thromboembolic events etc.).

ii. Patients with pre-existing diseases such as high blood pressure, diabetes, hyperlipidemia generally suffer of a worsening of these diseases which then become difficult to control and require a higher medication.

iii. In many instances patients develop newly diagnosed disease in the course of their COVID-19 infection, such as high blood pressure, diabetes, hyperlipidemia, which need to be continually followed up even after the patients recovers from COVID-19.

iv. Another chronic underlying diseases that are well controlled (solid cancers, hematologic diseases, autoimmune diseases, etc.) do not have much influence on the conversion of COVID-19 to severe form.

ISSN: 2574-1241

DOI: 10.26717/BJSTR.2021.39.006270

Youngah Choi. Biomed J Sci \& Tech Res

(c) (9) This work is licensed under Creative

Submission Link: https://biomedres.us/submit-manuscript.php v. Obesity is a risk factor for severe respiratory failure regardless of age or underlying disease.

\section{Clinical Course and Treatment of COVID-19 Disease}

i. Each person has a different incubation period (7-10 days) depending on the individual's immunity, the duration of the illness and the transition period of the corona virus test to negative (14-21 days). The clinical features and complications experienced by each person are different.

ii. Continued intake of medications to control existing chronic diseases.

iii. Popular treatment (fluid treatment, antibiotic treatment, antipyretic analgesic, and anti-inflammatory drugs, etc.) for symptom relief and normalization of test values, control of abnormal findings such as high fever, cough, sputum and pain by public medications is also helpful to overcome mild to moderate COVID-19 disease.

iv. Monoclonal Antibody (Regdanvimab) is effective in preventing progression to severe disease pattern when administered within 7 days of a diagnosed pneumonia (by CT scan) for patients under 60 years and in any case for patients older than 60 even when oxygen saturation is maintained above $95 \%$.

v. The antiviral drug(Veklury) has been proven effective when administered as early as possible in case of pneumonia(confirmation by CT scan) and an oxygen saturation level below $94 \%$.

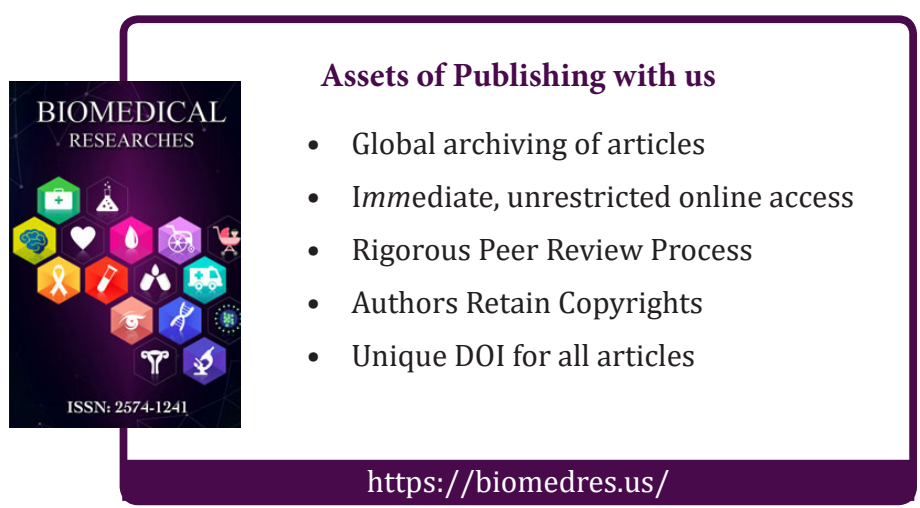

